\title{
MORFOLOGIA DE PLÂNTULAS E PLANTAS JOVENS DE 30 ESPÉCIES ARBÓREAS DE LEGUMINOSAE'
}

\author{
Denise Maria Trombert Oliveira ${ }^{2}$
}

\section{Recebido em 28/01/1999. Aceito em 16/08/1999}

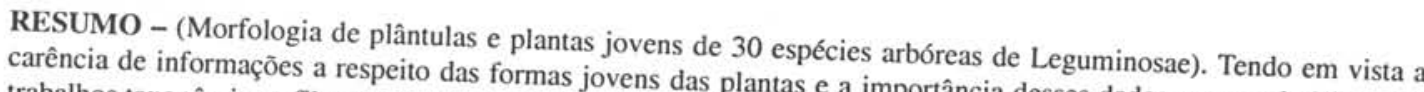
carência de informações a respeito das formas jovens das plantas e a importância desses dados como subsídios para 30 espécies arbóreas de Leguminosae, ocorrentes no Estado estudadas morfologicamente as plântulas e plantas jovens de úteis para a identificação das espécies selecionadas. $O$ desenvolvimento, Brasil, visando à apresentação de características diariamente, em germinador e em casa de vegetação. São apresentados das plântulas e plantas jovens foi acompanhado de catáfilos, época de diferenciação do primeiro eófilo e sua filotaxia, época da abscisĩo cotilodogia da plântula, número primeiro metáfilo, bem como a ocorrência de nodulação radicular. A análise das plântulas e plantas jovens da formação do a grande variação que existe nas Leguminosae. Nessa família, plântulas epígeo-foliáceas e epígeo-carnosas ocorrem em carnosos. Em Mimosoideae, 66,7\% das espécies prodas as plântulas se mostraram epígeas, $20 \%$ delas com cotilédones semi-hipógeas. As espécies de Faboideae apresentaram dois tântulas epígeo-foliáceas, 22,2\% epígeo-carnosas e 11,1\% em $45,5 \%$.
\end{abstract}

Palavras-chave - Leguminosae, morfologia, plântula, planta jovem

\begin{abstract}
Morphology of seedlings and saplings of 30 tree species of Leguminosae). Seedlings and saplings of due to the scarce information about seedlings and saplings besides studied morphologicaily. This study was also carried out phylogenetic and ecological studies. The seedling and sapling the importance of these data as a support for taxonomic, greenhouse. Seedling morphology, cataphyll number, first eophyll development were observed daily in a germinator and abscision time, first metaphyll differentiation and radicular eophyll differentiation time and their phyllotaxis, cotyledon seedling variation in Leguminosae. In this family, epigeal-foliaceous and epigeal-fled. The results showed the greatest the studied species. In Caesalpinioideae, all seedlings were epigeal, $20 \%$ of these seedlings heedlings occurred in $80 \%$ of Mimosoideae, $66.7 \%$ of the species had epigeal-foliaceous seedlings, $22.2 \%$ of these seedlings had fleshy cotyledons. In seedlings. Faboideae species exhibited two types of seedlings: $54.5 \%$ epigeal-fleshy and $11.1 \%$ semi-hypogeal
\end{abstract}

Key words - Leguminosae, morphology, seedling, sapling

\section{Introdução}

É reconhecida por todos a importância florística e econômica da família Leguminosae no Brasil. Mesmo sendo das raras famílias botânicas sobre as quais existem numerosos estudos morfológicos, dados sobre as fases juvenis são ainda escassos e pulverizados, muitas vezes aparecendo em trabalhos que abordam espécies de diversas familias (Compton 1912; Duke 1965; 1969; Ricardi et al. 1977, 1987).

\footnotetext{
Parte da tese de Doutorado da Autora. Auxílios CNPq, CAPES (PICD), FUS

$49^{\circ}$ Congresso Nacional de Botânica, Salvador, BA

2 Departamento de Botânica, IB, UNESP, C.Postal 510 ,
}

, CEP 18.618-000, Botucatu, SP, Brasil. e-mail: doliveira@laser.com.br 
Relatos de plântulas de leguminosas brasileiras podem ser encontrados em Silva et al. (1988), Lima (1989-90) e Oliveira \& Beltrati (1992).

As plântulas e plantas jovens apresentam grande fragilidade estrutural, estando sujeitas a danos diversos produzidos pelo pisoteio de animais, queda de ramos ou herbivoria. Desta forma, o período juvenil pode ser considerado o mais crítico do ciclo de vida de muitas espécies (Kozlowski 1971), uma vez que um fracasso dos processos adaptativos nesse estádio poderia, a longo prazo, levar a espécie à extinção (Amo-Rodríguez \& Gómez-Pompa 1976). Além de crítica, a fase de plântula é pouco conhecida. A capacidade de se reconhecerem, num dado momento, as plântulas e plantas jovens na mata pode ser de grande valor para se estabelecer a dinâmica de populações da mesma e o manejo silvicultural de muitas áreas semelhantes (Silva et al. 1988). Pode também ser útil para se reconhecer o estádio sucessional de uma vegetação, analisando-se índices de diversidade de espécies (Amo 1979; Torres 1985). Segundo $\mathrm{Ng}$ (1973), resultados sobre a velocidade de germinação de sementes, aliados aos dados de morfologia e desenvolvimento de plântulas são muito úteis nos trabalhos em viveiros e em pesquisas sobre armazenamento de sementes e regeneração de florestas.

Especialmente nas regiões neotropicais, o desmatamento indiscriminado já provocou a extinção de diversas espécies vegetais, colocando em risco numerosas outras. No ponto de vista de Garwood \& Humphries (1993), para reverter essa desastrosa tendência de desmatamento e extinção em regiões tropicais, é necessário habilidade para manejar e conservar os remanescentes florestais, constituindo áreas de proteção ambiental ou de manejo sustentável, restaurando-se, também, as áreas já devastadas.

Tendo em vista essas considerações, tornouse oportuna a realização do estudo comparativo de 30 espécies arbóreas de Leguminosae que ocorrem no Estado de São Paulo, as quais são freqüentes nos demais Estados da região sudeste e no sul do Brasil, algumas delas atingindo a região amazônica (Lorenzi 1992; 1998). No presente trabalho, são apresentadas informações sobre a morfologia de plântulas e plantas jovens das espécies selecionadas, objetivando prover subsídios para o reconhecimento destas espécies nas formações naturais e embasar futuros trabalhos taxonômicos, fillogenéticos e ecológicos.

\section{Material e métodos}

Para realizar o presente trabalho, foram coletadas sementes maduras das espécies a seguir listadas, que seguem os táxons propostos por Polhill \& Raven (1981) e estão acompanhadas de seus respectivos nomes comuns (Lorenzi 1992, 1998), acrescidas do número de registro no Herbarium Rioclarense (HRCB), pertencente ao Departamento de Botânica, Instituto de Biociências, UNESP, Rio Claro, e do local de coleta das sementes:

\section{Caesalpinioideae}

\section{Caesalpinieae}

Caesalpinia leiostachya Ducke (pau-ferro) - HRCB 26.508 - coletada no campus da UNESP, Rubião Júnior, Botucatu, SP;

Dimorphandra mollis Benth. (faveira, barbatimão-falso) - HRCB 26.501 - coletada em remanescentes de cerrado no município de Botucatu, SP;

Peltophorum dubium (Spreng.) Taub. (canafístula, farinha-seca) - HRCB 26.509 coletada no campus da UNESP, Rubião Júnior, Botucatu, SP;

Pterogyne nitens Tul. (amendoim, amendoim-bravo) - HRCB 26.498 - coletada no campus da UNESP, Fazenda Lageado, Botucatu, SP;

Schizolobium parahyba (Vell.) Blake (guapuruvu, ficheira) - HRCB 26.494 - coletada no campus da UNESP, Fazenda Lageado, Botucatu, SP;

Cassieae - coletadas no campus da UNESP, Rubião Júnior, Botucatu, SP;

Cassia ferruginea (Schrad.) Schrad. ex DC. (chuva-de-ouro) - HRCB 26.506;

Senna multijuga (Rich.) Irwin et Barn. (paucigarra) - HRCB 26.516;

\section{Cercideae}

Bauhinia forficata Link (pata-de-vaca, unhade-vaca, casco-de-vaca) - HRCB 26.515 - coletada 
na Reserva Florestal da Fazenda Edgardia, pertencente à UNESP, campus de Botucatu;

\section{Detarieae}

Copaifera langsdorffii Desf. (copaíba, óleode-copaíba) - HRCB 26.493 - coletada em remanescentes de mata no município de Botucatu, SP;

Hymenaea stilbocarpa Hayne (jatobá, jatá) - HRCB 26.504 - coletada no campus da UNESP, Fazenda Lageado, Botucatu, SP;

\section{Faboideae}

\section{Dalbergieae}

Centrolobium tomentosum Guill. ex Benth. (araribá, araribá-rosa) - HRCB 26.492 - coletada na Reserva Florestal da Fazenda Edgardia, pertencente à UNESP, campus de Botucatu;

Dalbergia nigra (Vell.) Fr. All. ex Benth. (jacarandá-da-bahia) - HRCB 13.373 - coletada no campus da UNESP, Rio Claro, SP;

Machaerium acutifolium Vog. (jacarandá-docampo, bico-de-pato) - HRCB 26.502 - coletada no campus da UNESP, Rubião Júnior, Botucatu, SP;

Platypodium elegans Vog. (amendoim-docampo) - HRCB 26.514 - coletada no campus da UNESP, Rio Claro, SP;

Tipuana tipu (Benth.) O. Kuntze (tipuana) HRCB 26.510 - coletada no campus da UNESP, Rubião Júnior, Botucatu, SP;

\section{Phaseoleae}

Erythrina speciosa Andrews (mulungu-dolitoral, eritrina-candelabro) - HRCB 26.511 coletada no campus da UNESP, Rubião Júnior, Botucatu, SP;

\section{Sophoreae}

Holocalyx balansae Mich. (alecrim-decampinas) - HRCB 26.497 - coletada no campus da UNESP, Fazenda Lageado, Botucatu, SP;

Sophora tomentosa. L. (sofora) - HRCB 17.928 - coletada em remanescente de mata em Picinguaba, SP;

\section{Swartzieae}

Swartzia langsdorffii Raddi (pacová-demacaco, jacarandá-banana) - HRCB 26.505 coletada no campus da UNESP, Fazenda Lageado, Botucatu, SP;

\section{Tephrosieae}

Lonchocarpus muehlbergianus Hassl. (embira-de-sapo) - HRCB 26.496 - coletada em remanescentes de mata no município de Botucatu, $\mathrm{SP}$;

Platycyamus regnellii Benth. (pau-pereira) - HRCB 26.499 - coletada no campus da UNESP, Fazenda Lageado, Botucatu, SP;

\section{Mimosoideae}

Acacieae

Acacia polyphylla DC. (monjoleiro) - HRCB 26.513 - coletada na Reserva Florestal da Fazenda Edgardia, pertencente à UNESP, campus de Botucatu;

\section{Ingeae}

Enterolobium contortisiliquum (Vell.) Morong (orelha-de-negro) - HRCB 26.507 coletada na Reserva Florestal da Fazenda Edgardia, pertencente à UNESP, campus de Botucatu;

Inga edulis Mart. (ingá-cipó, ingá-demacaco, ingá-de-metro, angá) - HRCB 26.518 coletada em indivíduos cultivados em Silvestre, Viçosa, MG;

Pithecellobium inopinathum Ducke (setecascas) - HRCB 26.495 - coletada no campus da UNESP, Fazenda Lageado, Botucatu, SP;

\section{Mimoseae}

Anadenanthera macrocarpa (Benth.) Brenan (angico, angico-vermelho) - HRCB 26.533 coletada no campus da UNESP, Rubião Júnior, Botucatu, SP;

Mimosa caeasalpiniaefolia Benth. (sabiá, sansão-do-campo) - HRCB 26.503 - coletada no campus da UNESP, Fazenda Lageado, Botucatu, $\mathrm{SP}$;

Piptadenia gonoacantha (Mart.) Macbr. (pau-jacaré) - HRCB 26.517 - coletada no campus da UNESP, Fazenda Lageado, Botucatu, SP;

Plathymenia foliolosa Benth. (vinhático) HRCB 26.519 - coletada no campus da Universidade Federal de Viçosa, MG;

Stryphnodendron adstringens (Mart.) Coville (barbatimão) - HRCB 26.500 - coletada em remanescentes de cerrado no município de Botucatu, SP. 
As amostras de sementes duras foram desinfestadas em água sanitária comercial a $20 \%$, por 15 minutos, sendo lavadas em água corrente; sementes de tegumento delgado permaneceram na solução por somente cinco minutos, seguindo-se a lavagem. Antes de se proceder à desinfestação, eliminaram-se das sementes todas as porções carnosas externas. Quando os frutos eram sâmaras de núcleo lenhoso (Centrolobium tomentosum, Platypodium elegans e Tipuana tipu), desinfestaram-se e semearam-se os núcleos seminíferos, sendo eliminadas as alas. Cada semente dura foi escarificada mecanicamente, utilizando-se lima.

Uma amostra de 50 sementes de cada espécie (cinco repetições de dez unidades cada) foi colocada em caixas tipo ger-box, entre folhas de papel de filtro umedecidas com água destilada em germinador (modelo NT708) a $25^{\circ} \mathrm{C} \pm 1$, sob iluminação fluorescente branca contínua (1.000 lux, luz do dia). Antes das plântulas atingirem o tamanho da caixa, foram transplantadas para sacos de polietileno próprios para produção de mudas, contendo mistura de terra e areia $(1: 1 \mathrm{p} / \mathrm{p})$, sendo mantidas em casa-de-vegetação recoberta por tela plástica, que produz sombreamento de $50 \%$, e regadas diariamente. Foram realizadas observações diárias, registrando-se o início da germinação (determinado pela emergência da raiz primária) e o paulatino desenvolvimento das plântulas e plantas jovens, até a formação dos primeiros metáfilos.

Concomitantemente, semeou-se outra amostra de 50 sementes de cada espécie em bandejas plásticas, contendo mistura de terra e areia (1:1 p/ p), sendo mantidas em casa-de-vegetação e regadas diariamente. As plântulas obtidas foram transplantadas para sacos de polietileno com substrato semelhante, sendo mantidas e acompanhadas em estufa de sombrite. As observações realizadas nesses indivíduos complementaram as realizadas naqueles obtidos em germinador. Foram consideradas germinadas as sementes que apresentaram hipocótilo ou epicótilo emergindo do solo, conforme o padrão da espécie.

A terminologia referente ao desenvolvimento das plântulas e plantas jovens baseou-se nos trabalhos de Duke (1965, 1969), Duke \& Polhill (1981) e em Miquel (1987). A definição do metáfilo das espécies estudadas foi feita com base na análise das plantas utilizadas como matrizes, bem como em informações da literatura (Burkart 1952; Lorenzi 1992).

\section{Resultados e discussão}

Os resultados sobre a morfologia das plântulas e a caracterização das plantas jovens de Caesalpinioideae, Faboideae e Mimosoideae encontram-se nas Tab. 1-3, respectivamente. Descrições detalhadas de cada espécie podem ser encontradas em Oliveira (1997).

Analisando plântulas de leguminosas, Gates (1951) referiu que as espécies de Caesalpinioideae e Mimosoideae pareciam ser todas epígeas, enquanto que, em Faboideae, algumas tribos, como Dalbergieae, eram epígeas, outras como Sophoreae eram hipógeas e que nas Phaseoleae ocorriam transições, apresentando tanto germinação epígea quanto hipógea.

Estudos posteriores ao de Gates (1951) confirmaram que as espécies de Caesalpinioideae e Mimosoideae são fundamentalmente epígeas, com cotilédones que podem ser foliáceos ou carnosos (Polhill et al. 1981).

Dentre as Caesalpinioideae, Duke \& Polhill (1981) reconheceram três grupos de plântulas: em Cercideae, as plântulas apresentam eófilos muito semelhantes aos metáfilos; em Detarieae e Amherstieae, quase sempre mostram cotilédones carnosos e, em Cassieae e Caesalpinieae, as plântulas são epígeas, com cotilédones foliáceos (Tab. 1). Na subfamília Mimosoideae, as plântulas seguem padrão semelhante.

Dentre as dez espécies de Caesalpinioideae analisadas neste trabalho, todas se mostraram epígeas, sendo $80 \%$ delas com cotilédones foliáceos. Cotilédones carnosos ocorreram exclusivamente nos representantes de Detarieae. Os três grupos referidos por Duke \& Polhill (1981) para as Caesalpinioideae foram amostrados neste trabalho: Bauhinia forficata (Cercidae) apresenta eófilos e metáfilos similares, distintos apenas pela presença de estípulas transformadas em espinhos; Copaifera langsdorffii e Hymenaea stilbocarpa (Detarieae) são epígeo-carnosas; e as espécies de Caesalpinieae (Caesalpinia leiostachya, Dimor- 
Tabela 1. Morfologia das plântulas e plantas jovens de espécies de Caesalpinioideae $(\mathrm{n}=100)$.

\begin{tabular}{|c|c|c|c|c|c|c|c|c|}
\hline Tribo / Espécie & $\begin{array}{c}\text { Tipo } \\
\text { de } \\
\text { Plântula } \\
\end{array}$ & $\begin{array}{c}\text { Número } \\
\text { de } \\
\text { catáfilos }\end{array}$ & $\begin{array}{c}\text { Diferenciação } \\
\text { do } 1^{2} \\
\text { eófilo } \\
\end{array}$ & $\begin{array}{c}\text { Filotaxia } \\
\text { do } 1^{2} \\
\text { eófilo } \\
\end{array}$ & $\begin{array}{c}\text { Número } \\
\text { de } \\
\text { eófilos } \\
\end{array}$ & $\begin{array}{l}\text { Abscisão } \\
\text { do } \\
\text { cotilédone }\end{array}$ & $\begin{array}{c}\text { Diferenciação } \\
\text { do } 1^{\Omega} \\
\text { metáfilo }\end{array}$ & $\begin{array}{l}\text { Nódulo } \\
\text { Radicular }^{\prime}\end{array}$ \\
\hline \multicolumn{9}{|c|}{ Caesalpinieae } \\
\hline Caesalpinia leiostachya & epígeo-foliácea & 0 & 25 dias & alterna & $04-06$ & 35 dias & 08 meses & - \\
\hline Dimorphandra mollis & epígeo-foliácea & 0 & 25 dias & oposta & $10-18$ & 40 dias & 10 meses & - \\
\hline Peltophorum dubium & epígeo-foliácea & 0 & 25 dias & oposta & $11-13$ & 40 dias & 07 meses & - \\
\hline Pterogyne nitens & epígeo-foliácea & 0 & 18 dias & alterna & $03-07$ & 30 dias & 05 meses & - \\
\hline $\begin{array}{l}\text { Schizolobium parahyba } \\
\text { Cassieae }\end{array}$ & epígeo-foliácea & 0 & 20 dias & oposta & $12-18$ & 30 dias & 07 meses & - \\
\hline \multicolumn{8}{|c|}{$\begin{array}{l}\text { Pterogyne nitens } \\
\text { Schizolobium parahyba epígeo-foliácea } \\
\text { Cassieae }\end{array}$} & - \\
\hline $\begin{array}{l}\text { Cassia ferruginea } \\
\text { Senna multijuga } \\
\text { Cercideae }\end{array}$ & $\begin{array}{l}\text { epígeo-foliácea } \\
\text { epígeo-foliácea }\end{array}$ & $\begin{array}{l}0 \\
0\end{array}$ & $\begin{array}{l}18 \text { dias } \\
20 \text { dias }\end{array}$ & alterna & $15-20$ & 45 dias & 08 meses & - \\
\hline $\begin{array}{l}\text { Cercideae } \\
\text { Bauhinia forficata } \\
\text { Detarieae }\end{array}$ & epígeo-foliácea & 0 & 22 dias & alterna & $02-03$ & 30 dias & 02 meses & - \\
\hline $\begin{array}{l}\text { Detarieae } \\
\text { Copaifera langsdorffii }\end{array}$ & epígeo-carnosa & 0 & 21 dias & oposta & $04-08$ & 28 dias & 05 meses & - \\
\hline Hymenaea stilbocarpa & epígeo-carnosa & 0 & 28 dias & alterna & 01 & 45 dias & 50 dias & - \\
\hline
\end{tabular}

1 - nódulos ausentes.

Tabela 2. Morfologia das plântulas e plantas jovens de espécies de Faboideae $(\mathrm{n}=100)$.

\begin{tabular}{|c|c|c|c|c|c|c|c|c|}
\hline Tribo / Espécie & $\begin{array}{cc}\text { Tipo } & \mathrm{N} \\
\text { de } & \\
\text { Plântula } & \mathrm{c} \\
\end{array}$ & $\begin{array}{c}\text { Número } \\
\text { de } \\
\text { catáfilos }\end{array}$ & $\begin{array}{c}\text { Diferenciação } \\
\text { do } 1^{2} \\
\text { eófilo } \\
\end{array}$ & $\begin{array}{c}\text { Filotaxia } \\
\text { do } 1^{\circ} \\
\text { eófilo } \\
\end{array}$ & $\begin{array}{c}\text { Número } \\
\text { de } \\
\text { eófilos }\end{array}$ & $\begin{array}{l}\text { Abscisão D } \\
\text { do } \\
\text { cotilédone } \\
\end{array}$ & $\begin{array}{c}\text { Diferenciação } \\
\text { do } 1^{2} \\
\text { metáfilo }\end{array}$ & $\begin{array}{c}\text { Nódulo } \\
\text { Radicular }^{1}\end{array}$ \\
\hline \multicolumn{9}{|c|}{ Dalbergieae } \\
\hline Centrolobium tomentosum & epígeo-carnosa & 0 & 31 dias & oposta & $08-13$ & 45 dias & 04 meses & + \\
\hline Dalbergia nigra & epígeo-carnosa & 0 & 20 dias & oposta & $05-07$ & 40 dias & 02 meses & + \\
\hline Machaerium acutifolium & epígeo-carnosa & 0 & 36 dias & oposta & $14-26$ & 45 dias & 10 meses & + \\
\hline Platypodium elegans & hipógea & $3-5$ & 30 dias & alterna & $05-09$ & 03 meses & 06 meses & + \\
\hline & epígeo-carnosa & 0 & 30 dias & alterna & $05-09$ & 45 dias & 05 meses & + \\
\hline $\begin{array}{l}\text { Phaseoleae } \\
\text { Erythrina speciosa } \\
\text { Sophoreae }\end{array}$ & epígeo-carnosa & 0 & 16 dias & oposta & 02 & 25 dias & 30 dias & + \\
\hline \multicolumn{2}{|l|}{$\begin{array}{l}\text { Erythrina speciosa } \\
\text { Sophoreae }\end{array}$} & & 45 dias & alterna & $05-07$ & 03 meses & 04 meses & - \\
\hline $\begin{array}{l}\text { Holocalyx balansae } \\
\text { Sophora tomentosa }\end{array}$ & hipógea & $3-5$ & 30 dias & alterna & $06-15$ & 04 meses & 08 meses & + \\
\hline $\begin{array}{l}\text { Swartzieae } \\
\text { Swartzia langsdorffii }\end{array}$ & hipógea & $3-5$ & 04 meses & alterna & $07-10$ & 06 meses & 13 meses & + \\
\hline \multicolumn{4}{|l|}{$\begin{array}{l}\text { Swartzia langsdorffu } \\
\text { Tephrosieae }\end{array}$} & oposta & $08-11$ & 02 meses & 06 meses & + \\
\hline $\begin{array}{l}\text { Lonchocarpus muehlbergiamus } \\
\text { Platycyamus regnellii }\end{array}$ & epígeo-carnosa & $\begin{array}{l}0 \\
0\end{array}$ & 38 dias & oposta & 02 & 50 dias & 02 meses & - \\
\hline
\end{tabular}

I + nódulos presentes; - nódulos ausentes.

phandra mollis, Peltophorum dubium, Pterogyne nitens e Schizolobium parahyba) e Cassieae (Cassia ferruginea e Senna multijuga) mostraramse sempre epígeo-foliáceas, concordando com o descrito pelos autores supra-citados.

Segundo Cowan \& Polhill (1981), em Detarieae formam-se geralmente eófilos opostos no primeiro nó, com tendências à formação de catáfilos. Das espécies estudadas, Copaifera langsdorffii forma eófilos opostos, mas não produz catáfilos. Já Hymenaea stilbocarpa forma o primeiro eófilo alterno e séssil, com dois grandes folíolos que se abrem para as laterais, dando a impressão de que são opostos.

Em Mimosoideae, 66,7\% das espécies analisadas mostraram-se epígeo-foliáceas, $22,2 \%$ epígeo-carnosas e $11,1 \%$ semi-hipógeas, estando estes dois últimos grupos representados exclusivamente pela tribo Ingeae (Tab. 3). 
Tabela 3. Morfologia das plântulas e plantas jovens de espécies de Mimosoideae $(n=100)$.

\begin{tabular}{|c|c|c|c|c|c|c|c|c|}
\hline Tribo/Espécie & $\begin{array}{c}\text { Tipo } \\
\text { de } \\
\text { Plântula }\end{array}$ & $\begin{array}{l}\text { Número I } \\
\text { de } \\
\text { catáfilos }\end{array}$ & $\begin{array}{c}\text { Diferenciação } \\
\text { do } 1^{2} \\
\text { eófilo } \\
\end{array}$ & $\begin{array}{c}\text { Filotaxia } \\
\text { do } 1^{\varrho} \\
\text { eófilo }\end{array}$ & $\begin{array}{c}\text { Número } \\
\text { de } \\
\text { éffilos }\end{array}$ & $\begin{array}{l}\text { Abscisão I } \\
\text { do } \\
\text { cotilédone }\end{array}$ & $\begin{array}{c}\text { Diferenciação } \\
\text { do } \mathbb{L}^{£} \\
\text { metáfilo }\end{array}$ & $\begin{array}{c}\text { Nódulo } \\
\text { Radicular }\end{array}$ \\
\hline \multicolumn{9}{|l|}{ Acacieae } \\
\hline $\begin{array}{l}\text { Acacia polyphylla } \\
\text { Ingeae }\end{array}$ & & oposta & $08-14$ & & 05 meses & - \\
\hline Enterolobium contortisiliquun & epígeo-carnosa & 0 & 26 dias & alterna & $06-10$ & 35 dias & 06 meses & + \\
\hline Inga edulis & semi-hipógea & 0 & 20 dias & oposta & $08-10$ & 02 meses & 06 meses & + \\
\hline $\begin{array}{l}\text { Pithecellobium inopinathum } \\
\text { Mimoseae }\end{array}$ & epígeo-carnosa & 0 & 20 dias & alterna & $06-11$. & 30 dias & 07 meses & + \\
\hline Anadenanthera macrocarpa & epígeo-foliácea & 0 & 13 dias & alterna & $06-12$ & 20 dias & 03 meses & + \\
\hline Mimosa caesalpiniaefolia & epígeo-foliácea & 0 & 12 dias & alterna & $04-05$ & 22 dias & 02 meses & + \\
\hline Piptadenia gonoacantha & epígeo-foliácea & 0 & 12 dias & alterna & $06-08$ & 20 dias & 03 meses & + \\
\hline Plathymenia foliolosa & epígeo-foliácea & 0 & 21 dias & oposta & $08-11$ & 35 dias & 05 meses & + \\
\hline Stryphnodendron adstringens & epígeo-foliácea & 0 & 18 dias & alterna & $06-09$ & 20 dias & 03 meses & + \\
\hline
\end{tabular}

'+ nódulos presentes; - nódulos ausentes.

Os dados obtidos neste trabalho concordam com a afirmativa de que as Leguminosae são principalmente epígeas, uma vez que $80 \%$ das espécies apresentaram este padrão. Desconsiderando as Faboideae, a germinação epígea ocorreu em $94,7 \%$ das espécies estudadas.

Em relação às plântulas de Faboideae, Duke \& Polhill (1981) consideram-nas extremamente variadas, especialmente nas tribos que abrangem espécies lenhosas, como são as englobadas por este trabalho. Segundo esses autores, em Tephrosieae e Phaseoleae, as plântulas podem ser fanerocotiledonares ou criptocotiledonares, mas sempre apresentam cotilédones carnosos.

Dentre as espécies estudadas de Faboideae, observaram-se dois tipos de plântulas: epígeocarnosas em $54,5 \%$ das espécies e hipógeas, em $45,5 \%$ (Tab. 2). As espécies de Sophoreae e Swartzieae se mostraram hipógeas. Em Phaseoleae, a única espécie estudada (Erythrina speciosa) apresentou plântula epígeo-carnosa. Em Dalbergieae e Tephrosieae ocorreram os dois tipos de plântulas, sendo que, na primeira tribo, dentre os representantes avaliados, somente Platypodium elegans é hipógea e, na segunda tribo, cada espécie estudada apresentou um tipo.

É interessante destacar a similaridade entre as plântulas e plantas jovens de Platycyamus regnellii (Tephrosieae) e Erythrina speciosa (Phaseoleae) que, excetuando-se características de tamanho, diferem somente pela presença de espinhos e nectários extra-florais em Erythrina. A despeito de existir tendência dos tipos de plântula se tornarem fixos nas tribos mais avançadas, ocasionalmente ocorrem reversões como em Phaseoleae, em que as plântulas passam de hipógeas a epígeas em algumas espécies (Polhill et al. 1981). Este fato explica a estreita correlação mencionada entre Erythrina speciosa e Platycyamus regnellii, a primeira integrante de Phaseoleae, tribo derivada diretamente de Tephrosieae, onde Platycyamus regnellii está enquadrada.

Uma característica das Leguminosae que merece destaque é a capacidade de nodulação radicular por Rhizobium, bactérias fixadoras de nitrogênio. Grande parte das espécies arbóreas nativas ainda carece de informação a respeito da capacidade de nodulação (Silva et al. 1988). Segundo Corby (1981), a nodulação é geral para as espécies de Mimosoideae e Faboideae, sendo incomum em Caesalpinioideae. Dentre as espécies estudadas neste projeto, $56,7 \%$ apresentaram nodulação por estirpes nativas de Rhizobium, sem que se fizesse inoculação. Nenhuma das espécies de Caesalpinioideae nodulou. Holocalyx balansae, Platycyamus regnellii e Acacia polyphylla são as únicas espécies de Faboideae e Mimosoideae que não formaram nódulos radiculares. Vários podem ser os motivos da ausência de nódulos nesses sistemas radiculares, além da incapacidade 
de nodulação. É possível que tenham ocorrido problemas de especificidade das estirpes do solo com essas espécies, porém o mais provável é que a nodulação seja tardia e, como as espécies só foram acompanhadas até a formação do primeiro metáfilo (durante quatro, dois e cinco meses, respectivamente) os nódulos não estavam formados e não puderam ser relatados.

Os dados apresentados sobre a morfologia das plântulas e plantas jovens demonstram inequivocamente a grande variação que existe na família Leguminosae, mesmo dentro das subfamílias. Indicam também a necessidade de novos estudos, que abordem maior número de espécies, de modo a se estabelecer amplo conhecimento sobre a família, especialmente abordando ambientes específicos.

\section{Agradecimentos}

À Profa. Dra. Celia Massa Beltrati, do Departamento de Botânica, IB, UNESP, Rio Claro, pela dedicada orientação deste trabalho; ao Biólogo José Flávio Cândido Júnior, de Botucatu, SP, pelo acompanhamento de todas as fases deste trabalho e pelas críticas aos originais.

\section{Referências bibliográficas}

Amo, S. 1979. Clave para plántulas y estados juveniles de especies primarias de una Selva Alta Perennifolia em Veracruz, México. Biotica 4(2): 59-108.

Amo-Rodríguez, S. \& Gómez-Pompa, A. 1976. Crescimiento de estados juveniles en plantas de Selva Tropical Alta Perennifolia. Pp.549-565. In: A. Gómez-Pompa; C. Vásquez-Yanes; S. Amo-Rodríguez \& A. C. Butanda (Eds.), Investigaciones sobre la regeneracion de selvas altas en Veracruz, México. Continental, México.

Burkart, A. 1952. Las leguminosas argentinas silvestres y cultivadas. 2.ed. Acme Agency, Buenos Aires.

Corby, H. D. L. 1981. The systematic value of leguminous root nodules. Pp.657-669. In: R. M. Polhill \& P. H. Raven (Eds.), Advances in legume systematics. Royal Botanic Gardens, Kew.

Compton, R. H. 1912. Investigation of the seedling structure in the Leguminosae. Journal of the Linnean Society of London, Botany 41: 1-122.

Cowan, R. S. \& Polhill, R. M. 1981. Tribe 4. Detarieae DC. (1825). Pp.117-134. In: R. M. Polhill \& P. H. Raven (Eds.), Advances in legume systematics. Royal Botanic Gardens, Kew.

Duke, J. A. 1965. Keys for the identification of seedlings of some proeminent woody species in eight forest types in
Puerto Rico. Annals of the Missouri Botanical Gardens 52(3): 314-350.

Duke, J. A. 1969. On tropical seedlings. I. Seeds, seedlings, systems and systematics. Annals of the Missouri Botanical Gardens 56(2): 125-161.

Duke, J. A. \& Polhill, R. M. 1981. Seedlings of Leguminosae. Pp. 941-949. In: R. M. Polhill \& P. H. Raven (Eds.), Advances in legume systematics. Royal Botanic Gardens, Kew.

Garwood, N. C. \& Humphries, C. J. 1993. Seedling diversity in the neotropics. NERC News 27: 20-23.

Gates, R. R. 1951. Epigeal germination in the Leguminosae. Botanical Gazette 113: 151-157.

Kozlowski, T. T. 1971. Growth and development of trees. v.1. Academic Press, New York.

Lima, H. C. 1989-90. Tribo Dalbergieae (Leguminosae Papilionoideae) - morfologia dos frutos, sementes e plântulas e sua aplicação na Sistemática. Arquivos do Jardim Botânico do Rio de Janeiro 30: 1-42.

Lorenzi, H. 1992. Árvores brasileiras: manual de identificação e cultivo de plantas arbóreas nativas do Brasil. Editora Plantarum, Nova Odessa.

Lorenzi, H. 1998. Árvores brasileiras: manual de identificação e cultivo de plantas arbóreas nativas do Brasil. v.2. Editora Plantarum, Nova Odessa.

Miquel, S. 1987. Morphologie fonctionelle de plantules d'especes forestières du Gabon. Bulletin du Museum National d'Histoire Naturelle, Section B, Adansonia 9(1): 101-121.

$\mathrm{Ng}$, F. S. P. 1973. Germination of fresh seeds of Malaysian trees. The Malaysian Forester 36(2): 54-65.

Oliveira, D. M. T. 1997. Análise morfológica comparativa de frutos, sementes, plântulas e plantas jovens de $\mathbf{3 0}$ espécies arbóreas de Fabaceae ocorrentes no Estado de São Paulo. Tese de Doutorado. UNESP, Rio Claro.

Oliveira, D. M. T. \& Beltrati, C. M. 1992. Morfologia e desenvolvimento das plântulas de Inga fagifolia e $I$. urugüensis. Turrialba 42: 306-313.

Polhill, R. M. \& Raven, P. H. (Eds.). 1981. Advances in legume systematics. Part 1. Royal Botanic Gardens, Kew.

Polhill, R. M.; Raven, P. H. \& Stirton, C. H. 1981. Evolution and Systematics of the Leguminosae. Pp.1-26. In: R. M. Polhill \& P. H. Raven (Eds.), Advances in legume systematics. Royal Botanic Gardens, Kew.

Ricardi, M.; Torres, F.; Hernández, C. \& Quintero, R. 1977. Morfologia de plantulas de arboles venezolanos. I. Revista Florestal Venezolana 27: 15-56.

Ricardi, M.; Hernandez, C. \& Torres, F. M. 1987. Morfologia de plantulas de arboles de los bosques del Estado Merida, Venezuela. Talleres Graficos Universitarios, Merida.

Silva, M. F.; Goldman, G. H.; Magalhães, F. M. \& Moreira, F. W. 1988. Germinação natural de 10 espécies arbóreas da Amazônia - I. Acta Amazonica 18(1-2): 9-26.

Torres, E. B. 1985. Identificacion de plantulas de algunas especies arboreas del bosque de Niebla. Perez-Arbelaezia 1(1): 39-95.

Torres, E. B. 1986. Identificacion de plantulas de algunas especies arboreas del bosque de Niebla. II Parte. PerezArbelaezia 1(2): 165-209. 